\section{Cerebrospinal Fluid, Hyposmia, and Dementia in Alzheimer Disease: Insights from Dynamic PET and a Hypothesis}

TO THE EDITOR: I read with great interest Dr. de Leon and colleagues' recent paper (1). Using dynamic PET techniques, they demonstrate elegantly that ventricular cerebrospinal fluid (CSF) clearance is significantly lower in patients with Alzheimer disease (AD) and that this correlates inversely with amyloid deposition. Nasal CSF egress is described in vivo for the first time and shown to be greatly reduced in patients with $\mathrm{AD}(1)$. Despite some minor technical limitations of the study, which they describe, it is hereby lauded as an excellent proof of concept that amyloid deposition impedes CSF circulation. Certain previous models linking impaired CSF turnover with the pathophysiology of AD may be thus corroborated $(2,3)$.

These findings are interesting and important for several reasons. Hyposmia is an extremely common symptom in $\mathrm{AD}$, experienced by nearly $100 \%$ of patients (4). Rhinencephalon and olfactory bulb amyloid distribution has previously been implicated. Cells of the human olfactory apparatus are replenished and replaced by a population of endogenous neural stem cells, and this neuroregenerative system has been shown to be disrupted in $\mathrm{AD}(5,6)$. Indeed, preventing this neuroregenerative impediment or enhancing neurogenesis is being investigated as a treatment strategy in $\mathrm{AD}$ (5). Neurogenesis in the brain is closely linked with the flow of CSF as new neurons migrate along CSF flow gradients (7). Amyloid plaques have been shown to disrupt the process of endogenous neurogenesis (5). It could be thus hypothesized that hyposmia in $\mathrm{AD}$ could be secondary to disturbances in CSF flow secondary to amyloid deposition, leading to impaired neurogenesis.

In addition to olfaction, endogenous neurogenesis in the hippocampus plays an important role in learning and memory (8). Taken together, I propose the hypothesis that amyloid deposition in $\mathrm{AD}$ impedes ventricular CSF drainage, which in turn impairs endogenous neuroregenerative mechanisms. Impaired neuroregeneration thus contributes to dementia, hyposmia, and neuronal loss in AD.

Although the suggestion that neurodegeneration may include an element of neuroregenerative failure is not new (9), the symptom combination of dementia and hyposmia applies to virtually all neurodegenerative diseases, albeit to different degrees (4). It would be interesting to evaluate whether CSF circulation could contribute to the pathophysiology of these neurodegenerative diseases using similar dynamic PET studies. Furthermore, whereas CSF diversion through ventriculoperitoneal shunting was not found to be efficacious in $\mathrm{AD}$ in a randomized study (10), it would be noteworthy to learn whether similar CSF circulation problems exist in normal pressure hydrocephalus (which is amenable to CSF diversion procedures). Additionally, the role of paravascular glymphatic pathways in the clearance of CSF remains to be elaborated. Further studies are indicated, and the authors are once again congratulated on an interesting paper.

\section{REFERENCES}

1. de Leon MJ, Li Y, Okamura N, et al. Cerebrospinal fluid clearance in Alzheimer disease measured with dynamic PET. J Nucl Med. 2017;58:1471-1476.

2. Tarasoff-Conway JM, Carare RO, Osorio RS, et al. Clearance systems in the brain: implications for Alzheimer disease. Nat Rev Neurol. 2015;11:457-470.
3. Ethell DW. Disruption of cerebrospinal fluid flow through the olfactory system may contribute to Alzheimer's disease pathogenesis. J Alzheimers Dis. 2014; 41:1021-1030.

4. Hawkes C. Olfaction in neurodegenerative disorder. Adv Otorhinolaryngol. 2006;63:133-151.

5. Ekonomou A, Savva GM, Brayne C, et al. Stage-specific changes in neurogenic and glial markers in Alzheimer's disease. Biol Psychiatry. 2015;77:711-719.

6. Lim DA, Alvarez-Buylla A. Adult neural stem cells stake their ground. Trends Neurosci. 2014;37:563-571.

7. Sawamoto K, Wichterle H, Gonzalez-Perez O, et al. New neurons follow the flow of cerebrospinal fluid in the adult brain. Science. 2006;311:629-632.

8. Lledo PM, Alonso M, Grubb MS. Adult neurogenesis and functional plasticity in neuronal circuits. Nat Rev Neurosci. 2006;7:179-193.

9. Armstrong RJ, Barker RA. Neurodegeneration: a failure of neuroregeneration? Lancet. 2001;358:1174-1176.

10. Silverberg GD, Mayo M, Saul T, Fellmann J, Carvalho J, McGuire D. Continuous CSF drainage in AD: results of a double-blind, randomized, placebo-controlled study. Neurology. 2008;71:202-209.

\author{
Ashwin Kumaria \\ Queen's Medical Centre \\ Nottingham, U.K. NG7 $2 U H$ \\ Email: ashwin.kumaria@doctors.org.uk
}

Published online Jan. 11, 2018.

DOI: 10.2967/jnumed.117.206888

REPLY: We are very pleased to read the letter submitted by Dr. Ashwin Kumaria to The Journal of Nuclear Medicine in response to our research paper (1). We share with the writer an excitement in the potential for a PET-based method to estimate the clearance of cerebrospinal fluid (CSF). As pointed out in Dr. Kumaria's letter, an impaired removal of protein waste may contribute to the development of many neurodegenerative diseases. This hypothesis is supported by an increasing body of preclinical evidence (2). The CSF clearance deficits we observed in the ventricle and superior nasal turbinates in our Alzheimer disease study are part of the glymphatic pathway, a CSF and interstitial fluid (ISF) communication pathway largely designed for waste removal from the brain.

Rodent model studies have identified glymphatic pathway deficits in transgenic Alzheimer mice, in cerebrovascular disease, and in brain trauma models. Animal studies have also shown the modifiability of the glymphatic pathway under experimental sleep and exercise conditions (3).

Looking forward, Dr. Kumaria points out promising areas of investigation in humans. We offer that another poorly understood area worthy of study is the contribution of the glymphatic system to the presentation of antigens and immune cells to the brain and to their clearance out to the periphery.

Overall, we expect that future functional longitudinal human imaging studies will directly test the hypothesized causal and modifiable relationships between brain CSF and ISF clearance and the accumulation of misfolded proteins, thus creating new therapeutic possibilities for neurodegenerative diseases such as Alzheimer disease.

\section{REFERENCES}

1. de Leon MJ, Li Y, Okamura N, et al. Cerebrospinal fluid clearance in Alzheimer disease measured with dynamic PET. J Nucl Med. 2017;58:1471-1476.

2. Tarasoff-Conway JM, Carare RO, Osorio RS, et al. Clearance systems in the brain-implications for Alzheimer disease. Nat Rev Neurol. 2015;11:457-470. 